\title{
Further Examples of Explicit Krein Representations of Certain Subordinators
}

By

\author{
Catherine Donati-Martin* and Marc YoR**
}

\begin{abstract}
In a previous paper [1], we have shown that the gamma subordinators may be represented as inverse local times of certain diffusions. In the present paper, we give such representations for other subordinators whose Lévy densities are of the form $\frac{\mathcal{C}}{(\sinh (y))^{\gamma}}, 0<\gamma<2$, and the more general family obtained from those by exponential tilting. These densities are closely linked with those of the inverse local times of the squared radial Ornstein-Uhlenbeck processes.
\end{abstract}

\section{$\S 1$. Aim of the Paper and Summary of [1]}

\section{$\S 1.1$}

In this paper, we continue the program started in [1], that is to represent as many subordinators $\left(\mathcal{S}_{\ell}, \ell \geq 0\right)$, i.e. increasing Lévy processes started at 0 , as possible as inverse local times $\left(\tau_{\ell}, \ell \geq 0\right)$ of some particular $\mathbb{R}_{+}$-valued diffusion $\left(X_{t}\right)$, such that 0 is regular for itself, relatively to $X$. More precisely, assume that

$$
E\left[\exp \left(-\lambda \mathcal{S}_{\ell}\right)\right]=\exp (-\ell \Psi(\lambda)), \quad \lambda, l \geq 0,
$$

where $\Psi(\lambda)=\int_{0}^{\infty} \nu(d y)\left(1-e^{-\lambda y}\right)$. If the Lévy measure $\nu(d y)$ of $\left(\mathcal{S}_{\ell}, \ell \geq 0\right)$ is of the form

$$
\nu(d y)=h(y) d y, \quad \text { with } \quad h(y)=\int_{0}^{\infty} d \sigma(x) e^{-x y},
$$

Communicated by Y. Takahashi. Received November 15, 2005. Revised April 4, 2006. 2000 Mathematics Subject Classification(s): 60G51, 60J55.

* Laboratoire de Probabilités et Modèles Aléatoires, Université Paris VI et VII, 4 Place Jussieu - Case 188, F-75252 Paris Cedex 05.

** Laboratoire de Probabilités et Modèles Aléatoires, Université Paris VI et VII, 4 Place Jussieu - Case 188, F-75252 Paris Cedex 05 - Institut Universitaire de France.

(c) 2007 Research Institute for Mathematical Sciences, Kyoto University. All rights reserved. 
for some positive $\sigma$-finite measure $\sigma(d x)$ on $\mathbb{R}_{+}$, then, it is known, as a consequence of Krein's theory (cf. Knight [5], Kotani-Watanabe [6]), that there exists a unique diffusion $\left(X_{t}\right)$ taking values in $\mathbb{R}_{+}$whose inverse local time $\left(\tau_{\ell}, \ell \geq 0\right)$ at 0 is distributed as $\left(\mathcal{S}_{\ell}, \ell \geq 0\right)$. Finding $X$ when $\nu$ is given is called (here) Krein representation problem.

In our paper [1], we could fill in the following:

\section{Table 1}

\begin{tabular}{|c|c|c|}
\hline$h(y)$ & Generator of $\left(X_{t}\right)$ & Distribution \\
\hline $\begin{array}{c}\frac{\mathcal{C}}{y^{\alpha+1}} \\
(0<\alpha<1)\end{array}$ & $\mathcal{L}_{-\alpha}=\frac{1}{2} \frac{d^{2}}{d x^{2}}+\frac{\delta-1}{2 x} \frac{d}{d x} ; \quad \delta=2(1-\alpha)$ & $P^{\delta}$ \\
\hline $\begin{array}{l}\text { (2) } \frac{\mathcal{C}}{y^{\alpha+1}} e^{-\mu y} \\
(0<\alpha<1 ; \mu>0)\end{array}$ & $\begin{array}{l}\mathcal{L}_{-\alpha}^{\mu \downarrow}=\mathcal{L}_{-\alpha}+\sqrt{2 \mu} \frac{\widehat{K}_{\alpha}^{\prime}(\sqrt{2 \mu} x)}{\widehat{K}_{\alpha}(\sqrt{2 \mu} x)} \frac{d}{d x} \\
\text { where } \widehat{K}_{\alpha}(y)=y^{\alpha} K_{\alpha}(y), y>0\end{array}$ & $P^{\delta ; \mu \downarrow}$ \\
\hline $\begin{array}{r}\quad \frac{\mathcal{C}}{y} e^{-\mu y} \\
(\mu>0)\end{array}$ & $\mathcal{L}_{0}^{\mu \downarrow}=\frac{1}{2} \frac{d^{2}}{d x^{2}}+\left(\frac{1}{2 x}+\sqrt{2 \mu} \frac{K_{0}^{\prime}}{K_{0}}(\sqrt{2 \mu} x)\right) \frac{d}{d x}$ & $P^{2 ; \mu \downarrow}$ \\
\hline
\end{tabular}

The measures $\sigma_{i}(i=1,2,3)$ representing $h$ in (1), (2), (3) via formula (1.1) are

$\sigma_{1}(d x)=\frac{\mathcal{C}}{\Gamma(\alpha+1)} x^{\alpha} d x ; \sigma_{2}(d x)=\frac{\mathcal{C}}{\Gamma(\alpha+1)}(x-\mu)_{+}^{\alpha} d x ; \sigma_{3}(d x)=\mathcal{C} 1_{(x \geq \mu)} d x$.

Before we give some details about this Table, we explain our method of finding diffusions which solve some Krein representation problems.

\section{$\S 1.2$ Krein problem and the construction of associated diffusions}

Let $\left(X_{t}\right)_{t \geq 0}$ be an $\mathbb{R}_{+}$-valued diffusion, with family of laws $\left(P_{x}\right)_{x \in \mathbb{R}_{+}}$. We assume that 0 is regular, hence $X$ admits a local time at 0 , which we denote by $\left(L_{t}, t \geq 0\right)$. We use the notation

$$
\tau_{\ell}=\inf \left\{t: L_{t}>\ell\right\}, \quad \ell \geq 0,
$$


for the corresponding inverse local time at $0, \nu(d y)$ for its Lévy measure and $(\Psi(\theta), \theta \geq 0)$ for its Lévy exponent. We look for a new Markovian family $\left(\tilde{P}_{x}\right)_{x \in \mathbb{R}_{+}}$such that

$$
\left.\tilde{P}_{0}\right|_{\mathcal{F}_{\tau_{l}}}=\left.\exp \left(\Psi(\theta) l-\theta \tau_{l}\right) \cdot P_{0}\right|_{\mathcal{F}_{\tau_{l}}} .
$$

Since the process $\left(\exp \left(\Psi(\theta) L_{t}-\theta t\right), t \geq 0\right)$, which occurs in $(1.2)$, is not a $\left(\mathcal{F}_{t}\right)$ martingale, it seems natural to look for a function $(\varphi(x) ; x \geq 0)$ such that

$$
\left.\tilde{P}_{x}\right|_{\mathcal{F}_{t}}=\frac{\varphi\left(X_{t}\right)}{\varphi(x)} \exp \left(\Psi(\theta) L_{t}-\theta t\right) .\left.P_{x}\right|_{\mathcal{F}_{t}} .
$$

In particular, if $T_{0}(X)$ denotes the first hitting time of 0 by $X$, then,

$$
\frac{\varphi\left(X_{t \wedge T_{0}}\right)}{\varphi(x)} \exp \left(-\theta\left(t \wedge T_{0}\right)\right)
$$

is a $\left(P_{x}, \mathcal{F}_{t}\right)$ martingale, and assuming $\varphi$ to be decreasing, Doob's optional stopping theorem implies

$$
\varphi(x)=\varphi_{\theta \downarrow}(x):=E_{x}\left[\exp \left(-\theta T_{0}(X)\right)\right]
$$

(we have normalized $\varphi$ so that $\varphi(0)=1$ ). Thus, we are led naturally to consider the diffusion, denoted by $\left(X_{t}^{\theta \downarrow}, t \geq 0\right)$, with laws $\left(P_{x}^{\theta \downarrow}, x \geq 0\right)$, such that

$$
\left.P_{x}^{\theta \downarrow}\right|_{\mathcal{F}_{t}}=\left.\frac{\varphi_{\theta \downarrow}\left(X_{t}\right)}{\varphi_{\theta \downarrow}(x)} \exp \left(\Psi(\theta) L_{t}-\theta t\right) \cdot P_{x}\right|_{\mathcal{F}_{t}} .
$$

As follows clearly from the starting point (1.2) of our method, the inverse local time $\left(\tau_{\ell}, \ell \geq 0\right)$ of $\left(X_{t}^{\theta \downarrow}, t \geq 0\right)$ satisfies

$$
E_{0}^{\theta \downarrow}\left(\exp \left(-\lambda \tau_{\ell}\right)\right)=\exp (-\ell(\Psi(\lambda+\theta)-\Psi(\theta)))
$$

i.e. this inverse local time is the $\theta$-Esscher transform of $\left(\tau_{\ell}, \ell \geq 0\right)$ under $P_{0}$, or equivalently its Lévy measure is $e^{-\theta y} \nu(d y)$.

It is also noteworthy that, under some adequate restriction of their domains, the infinitesimal generators $\mathcal{L}^{\theta \downarrow}$ and $\mathcal{L}$ are related by

$$
\mathcal{L}^{\theta \downarrow}=\mathcal{L}+\frac{d}{d x}\left(\log \left(\varphi_{\theta \downarrow}(x)\right)\right) \cdot \frac{d}{d x},
$$

assuming for simplicity that

$$
\mathcal{L}=\frac{1}{2} \frac{d^{2}}{d x^{2}}+b(x) \frac{d}{d x} .
$$




\section{$\$ 1.3$ Details of Table 1}

In fact, the result for the first row goes back at least to MolchanovOstrovski [8]. The result for the second row is deduced from that in the first row with the help of the above discussion. Finally, the result for the third row was deduced by letting $\alpha \rightarrow 0$ in the second row, while taking care of the choice $^{1}$ of the local times made for $\mathcal{L}_{-\alpha}^{\mu \downarrow}$. A compendium of choices of local times for Bessel-like diffusions is made in [2].

\section{$\S 1.4$}

In the present paper, we wish to complete the preceding Table 1, by considering the family of Lévy measures on $\mathbb{R}_{+}$, which is indexed by three parameters, as follows:

$$
\nu_{\mu, \alpha, k}(d y)=\mathcal{C}\left(\frac{\mu}{\sinh (\mu y)}\right)^{\alpha+1} \exp (\mu k y) d y
$$

Let us discuss about the role of these parameters and some necessary conditions so that (1.5) is a Lévy measure. The "true" parameters are $\mu>0$, $k$, and $\alpha$. As before, $\mathcal{C}$ is simply there to ensure an additional degree of freedom, if necessary. In order that $\nu_{\mu, \alpha, k}(d y)$ be a Lévy measure, it must satisfy

$$
\int_{0}^{\infty}(x \wedge 1) \nu_{\mu, \alpha, k}(d x)<\infty, \quad \text { that is } \quad 0 \leq \alpha<1 \text { and } k<1+\alpha .
$$

We now recall, from Pitman-Yor ([11] formulae (16), p. 276) that, if $Q_{z}^{\delta, \mu}$, $0<\delta \equiv 2(1-\alpha)<2$ and $\mu>0$, denotes the distribution of the squared radial Ornstein-Uhlenbeck process with "dimension" $\delta$ and parameter $\mu$, started from $z$, i.e. the solution of

$$
d Z_{t}=2 \sqrt{Z_{t}} d B_{t}+\left(\delta-2 \mu Z_{t}\right) d t, \quad Z_{t} \geq 0, Z_{0}=z,
$$

for some Brownian motion $\left(B_{t}, t \geq 0\right)$. Then, under $Q_{0}^{\delta, \mu}$, the inverse local time $\left(\tau_{\ell}, \ell \geq 0\right)$ admits as its Lévy measure

$$
\mathcal{C}\left(\frac{\mu}{\sinh (\mu y)}\right)^{\alpha+1} \exp \left(\mu \frac{\delta}{2} y\right) d y
$$

which is a particular case of $(1.5)$, with $k=\delta / 2=(1-\alpha)$.

\footnotetext{
${ }^{1}$ As is well-known, the local time in a standard Markovian set up, at a given level, is unique up to a multiplicative constant, which for our studies, needs to be chosen carefully.
} 
In the next section, we shall show, essentially with the help of the recipe (1.4), how to construct a diffusion, indexed by the three parameters $(\alpha, \mu, k)$, which solves the Krein representation problem for $\nu_{\mu, \alpha, k}$.

\section{$\S 1.5$}

Among the new diffusions we are finding as solutions of Krein's problem, some are related by time change to the diffusions we found in [1] . We first discovered this relationship by applying the analytical identity

$$
W_{0, \beta}(z)=\sqrt{\frac{z}{\pi}} K_{\beta}\left(\frac{z}{2}\right)
$$

between $W_{0, \beta}$, the Whittaker function with parameters $(0, \beta)$, and $K_{\beta}$, the Bessel-Macdonald function with parameter $\beta$ (see the Appendix where we also refer to Lebedev [7] for definitions and properties of the special functions involved). Thus, a part of our present discussion may be considered as giving a probabilistic interpretation to (1.7).

We also develop a similar discussion for the analytical identity

$$
M_{0, \beta}(z)=4^{\beta} \Gamma(\beta+1) \sqrt{z} I_{\beta}\left(\frac{z}{2}\right)
$$

between the Whittaker function $M_{0, \beta}$ and the modified Bessel function $I_{\beta}$.

\section{§2. Solving Krein's Problem for $\nu_{\mu, \alpha, k}$}

\section{$\S 2.1$}

We take up the notation in Subsection 1.3; in fact, it is more convenient to consider the family of radial Ornstein-Uhlenbeck processes (and not their squares), which we shall denote as $\left(R^{\delta, \mu}(t), t \geq 0\right)$, and their laws $\left(P_{r}^{\delta, \mu}\right.$, $r \geq 0$ ). It will be helpful, for the sequel, to have the following formula at hand for the infinitesimal generator $\mathcal{L}_{-\alpha, \mu}$ of $R^{\delta, \mu}$ :

$$
\mathcal{L}_{-\alpha, \mu}=\frac{1}{2} \frac{d^{2}}{d x^{2}}+\left(\frac{\delta-1}{2 x}-\mu x\right) \frac{d}{d x} .
$$

It is well-known (see, e.g., Pitman-Yor [9], p. 454, formula (6.b)) that there is the relationship

$$
R^{\delta, \mu}(t)=e^{-\mu t} R^{\delta}\left(\frac{e^{2 \mu t}-1}{2 \mu}\right), \quad t \geq 0,
$$


where, on the RHS, $\left(R^{\delta}(u), u \geq 0\right)$ denotes a $\delta$-dimensional Bessel process. Thus, we obtain

$$
\left(\frac{e^{2 \mu T_{0}}-1}{2 \mu} ; P_{x}^{\delta, \mu}\right) \underset{(a)}{\stackrel{(\text { law })}{=}}\left(T_{0} ; P_{x}^{\delta}\right) \stackrel{(\text { law })}{=} \frac{x^{2}}{(b)}
$$

where, on the RHS, $\gamma_{\alpha}$ denotes a gamma variable with parameter $\alpha=1-\delta / 2$. (a) follows from (2.2), while (b) is well-known and goes back to Getoor [3]. See, e.g., Yor [13], for some variants...

We thus deduce the following formula from (2.3) with the help of elementary computations: for $-2 \alpha \mu<\theta$,

$$
\begin{aligned}
E_{x}^{\delta, \mu}\left(\exp \left(-\theta T_{0}\right)\right) & \\
\quad= & \frac{1}{\Gamma(\alpha)\left(\mu x^{2}\right)^{\theta / 2 \mu}} \int_{0}^{\infty} \frac{t^{\alpha-1+\theta / 2 \mu} e^{-t}}{\left(1+\frac{t}{\mu x^{2}}\right)^{\theta / 2 \mu}} d t \\
& =\frac{\Gamma(\alpha+\theta / 2 \mu)}{\Gamma(\alpha)}\left(\mu x^{2}\right)^{(\alpha-1) / 2} e^{\mu x^{2} / 2} W_{(1-\alpha-\theta / \mu) / 2, \alpha / 2}\left(\mu x^{2}\right),
\end{aligned}
$$

where $W_{a, b}$ denotes the Whittaker function with parameters $(a, b)$. See [7, p. 279] for the integral representation of $W_{a, b}$.

We now write

$$
\begin{aligned}
\nu_{\mu, \alpha, k}(d y) & =\mathcal{C}\left(\frac{\mu}{\sinh (\mu y)}\right)^{\alpha+1} \exp (\mu k y) d y \\
& \equiv \mathcal{C}\left(\frac{\mu}{\sinh (\mu y)}\right)^{\alpha+1} \exp \left(\mu \frac{\delta}{2} y\right) \exp (-\theta y) d y
\end{aligned}
$$

where $\theta=\mu\left(\frac{\delta}{2}-k\right), k<1+\alpha$. According to the preceding computation (2.4), we now find that the diffusion with infinitesimal generator

$$
\mathcal{L}_{-\alpha, \mu}^{\theta \downarrow} \equiv \mathcal{L}_{-\alpha, \mu}+\frac{d}{d x} \log \left\{\left(\mu x^{2}\right)^{(\alpha-1) / 2} e^{\mu x^{2} / 2} W_{(1-\alpha-\theta / \mu) / 2, \alpha / 2}\left(\mu x^{2}\right)\right\} \cdot \frac{d}{d x}
$$

solves Krein's representation problem for $\nu_{\mu, \alpha, k}$. We note in fact that $(1-\alpha-\theta / \mu) / 2=k / 2$, hence $W_{(1-\alpha-\theta / \mu) / 2, \alpha / 2}(\xi) \equiv W_{k / 2, \alpha / 2}(\xi)$.

The case where $k=0$ is particularly interesting, since on one hand 


$$
\nu_{\mu, \alpha, 0}(d y)=\mathcal{C}\left(\frac{\mu}{\sinh (\mu y)}\right)^{\alpha+1} d y
$$

and on the other hand (see Appendix)

$$
W_{0, \alpha / 2}(\xi)=\sqrt{\frac{\xi}{\pi}} K_{\alpha / 2}\left(\frac{\xi}{2}\right)
$$

so that the diffusion which solves Krein's representation problem for $\nu_{\mu, \alpha, 0}(d y)$ is the solution to

$$
d X_{t}=d B_{t}+\left[\frac{\delta-1}{2 X_{t}}+\mu X_{t}\left(\frac{\widehat{K}_{\alpha / 2}^{\prime}}{\widehat{K}_{\alpha / 2}}\right)\left(\mu \frac{X_{t}^{2}}{2}\right)\right] d t
$$

Here, we need to give some details about this computation:

a) We deduce from formula (2.5), in the particular case $k=0$, i.e. $\theta=\delta \mu / 2$ that

$$
\mathcal{L}_{-\alpha, \mu}^{\theta \downarrow}=\mathcal{L}_{-\alpha, \mu}+\left(\frac{\alpha}{x}+\mu x+\mu x \frac{K_{\alpha / 2}^{\prime}}{K_{\alpha / 2}}\left(\mu \frac{x^{2}}{2}\right)\right) \cdot \frac{d}{d x}
$$

with the help of formula (2.6).

b) Now, trivially

$$
\mathcal{L}_{-\alpha, \mu}+\mu x \frac{d}{d x}=\mathcal{L}_{-\alpha}
$$

and

$$
\frac{\alpha}{x}+\mu x \frac{K_{\alpha / 2}^{\prime}}{K_{\alpha / 2}}\left(\mu \frac{x^{2}}{2}\right)=\mu x \frac{\widehat{K}_{\alpha / 2}^{\prime}}{\widehat{K}_{\alpha / 2}}\left(\mu \frac{x^{2}}{2}\right)
$$

which translates into the stochastic differential equation form (2.7).

To summarize the above and as an introduction to the next discussion in Subsection 2.2 , we write down 
Table 2

\begin{tabular}{c|c|c}
$h(y)$ & Generator of $\left(X_{t}\right)$ & Distribution \\
\hline $\begin{array}{c}\frac{\mathcal{C}}{y^{\alpha+1}} e^{-\mu y} \\
(0<\alpha<1 ; \mu>0)\end{array}$ & $\mathcal{L}_{-\alpha}^{\mu \downarrow}=\mathcal{L}_{-\alpha}+\sqrt{2 \mu} \frac{\widehat{K}_{\alpha}^{\prime}}{\widehat{K}_{\alpha}}(\sqrt{2 \mu} x) \frac{d}{d x}$ & $\begin{array}{c}P^{\delta ; \mu \downarrow} \\
(\delta=2(1-\alpha))\end{array}$ \\
\hline $\begin{array}{c}\mathcal{C}\left(\frac{\mu}{\sinh (\mu y)}\right)^{\alpha+1} e^{\mu \frac{\delta}{2} y} \\
(0<\delta=2(1-\alpha)<2 ; \mu>0)\end{array}$ & $\mathcal{L}_{-\alpha, \mu} \equiv \mathcal{L}_{-\alpha}-\mu x \frac{d}{d x}$ & $P^{\delta, \mu}$ \\
\hline $\mathcal{C}\left(\frac{\mu}{\sinh (\mu y)}\right)^{\alpha+1}$ & $\mathcal{L}_{-\alpha, \mu}^{\theta \downarrow} \equiv \mathcal{L}_{-\alpha}+\mu x \frac{\widehat{K}_{\alpha / 2}^{\prime}}{\widehat{K}_{\alpha / 2}}\left(\mu \frac{x^{2}}{2}\right) \frac{d}{d x}$ & $P^{\delta, \mu ; \frac{\delta \mu}{2} \downarrow}$ \\
$(0<\alpha<1 ; \mu>0)$ & $\left(\theta=\frac{\delta \mu}{2}\right)$ &
\end{tabular}

The first row is simply taken from Table 1 (second row there). As said above, the second row here follows from Pitman-Yor [11]. In the third row, we have written $\mathcal{L}_{-\alpha, \mu}^{\theta \downarrow}$ for the infinitesimal generator of the process which is defined as the radial Ornstein-Uhlenbeck process with dimension $\delta=2(1-\alpha)$ and drift parameter $(-\mu)$, pushed downwards with parameter $\theta=\delta \mu / 2$. That this infinitesimal generator may be expressed in terms of $\widehat{K}_{\alpha / 2}$ has already been discussed after (2.7). Another argument is given in the next Subsection 2.2.

\section{$\S 2.2$}

We shall now prove a remarkable relationship between the two families of diffusions whose infinitesimal generators are found in Table 2. This relationship explains precisely why (Row 1) may be deduced from (Row 2), and vice-versa.

Proposition 2.1. $\quad$ Let $0<\alpha<1$ and $\delta=2(1-\alpha)$. The following relationship holds with $\theta=\delta \mu / 2$,

$$
X_{-\alpha, \mu ; \theta \downarrow}^{2}(t)=X_{-\frac{\alpha}{2} ; \frac{\mu^{2}}{8} \downarrow}\left(4 \int_{0}^{t} X_{-\alpha, \mu ; \theta \downarrow}^{2}(u) d u\right),
$$


meaning that, starting from $X \equiv X_{-\alpha, \mu ; \theta \downarrow}$ on the LHS, there exists $\left(X_{-\frac{\alpha}{2} ; \frac{\mu^{2}}{8} \downarrow}(u), u \geq 0\right)$ such that the relationship (2.8) holds.

Comment about our notation. In formula (2.8), and possibly several times below, we have written $X_{i ; \theta \downarrow}$, etc... instead of $X_{i}^{\theta \downarrow}$, for some index $i$. It seemed more appropriate here, because of the power 2 which occurs on both sides of (2.8). There should be no confusion for the reader between the different diffusions $X_{i, \theta}$ and $X_{i ; \theta \downarrow}$.

Proof. We start from the stochastic differential equation satisfied by $\left(X_{-\alpha, \mu ; \theta \downarrow}(t), t \geq 0\right)$ as described implicitly in Row 2 of Table 2. Taking squares, we obtain

$$
X_{t}^{2}=x^{2}+2 \int_{0}^{t} X_{s} d B_{s}+\delta t+2 \int_{0}^{t}\left(\mu X_{s}^{2}\right) \frac{\widehat{K}_{\alpha / 2}^{\prime}}{\widehat{K}_{\alpha / 2}}\left(\mu \frac{X_{s}^{2}}{2}\right) d s .
$$

We now define $\left(Y_{u} \equiv Y(u), u \geq 0\right)$ via

$$
X_{t}^{2}=Y\left(4 \int_{0}^{t} X_{s}^{2} d s\right), \quad t \geq 0
$$

and find that $Y$ satisfies

$$
Y_{u}=x^{2}+\beta_{u}+\left(\frac{\delta}{4}\right) \int_{0}^{u} \frac{d s}{Y_{s}}+\frac{1}{2} \int_{0}^{u} \mu \frac{\widehat{K}_{\alpha / 2}^{\prime}}{\widehat{K}_{\alpha / 2}}\left(\frac{\mu}{2} Y_{s}\right) d s
$$

for some Brownian motion $\left(\beta_{u}\right)$. Since $\delta / 4=(\widehat{\delta}-1) / 2$, with $\widehat{\delta}=2-\alpha=$ $2(1-\alpha / 2)$, it now appears that $\left(Y_{u}, u \geq 0\right)$ is precisely the diffusion with infinitesimal generator $\mathcal{L}_{-\alpha / 2}^{\nu \downarrow}$, with $\sqrt{2 \nu}=\mu / 2$, i.e. $\nu=\mu^{2} / 8$.

Let us note that the proof we have just given for the Proposition relies upon the identification of the infinitesimal generator of the diffusion $X_{-\alpha, \mu ;(\delta \mu / 2) \downarrow}$ as given in Table 2. This identification was obtained from an analytical identity between $W_{0, \text {. }}$ and $K_{\text {. }}$ ( (see formula $(2.6)$ ).

We now explain and prove the Proposition without relying on such identities, but rather on absolute continuity relationships between the different laws involved. We now find it a little more convenient to refer to the laws $\left\{Q_{z}^{\delta, \mu}\right\}$ and the main absolute continuity result we need is

$$
\left.Q_{z}^{\delta, \mu}\right|_{\mathcal{F}_{t}}=\left.\exp \left(-\frac{\mu}{2}\left(Z_{t}-\delta t-z\right)-\frac{\mu^{2}}{2} \int_{0}^{t} Z_{s} d s\right) \cdot Q_{z}^{\delta}\right|_{\mathcal{F}_{t}} .
$$

Here, $\left(Z_{t}, t \geq 0\right)$ denotes the coordinate process on the canonical space $C\left(\mathbb{R}_{+}\right.$, $\left.\mathbb{R}_{+}\right)$. We now combine this relation (2.9) with that of the "push downwards" 
with parameter $\theta$, so that, with notations which we shall explain after writing the formula

$$
\begin{aligned}
\left.Q_{z}^{\delta, \mu ; \theta \downarrow}\right|_{\mathcal{F}_{t}}= & \frac{\varphi_{\theta \downarrow}\left(Z_{t}\right)}{\varphi_{\theta \downarrow}(z)} \exp \left(\Psi(\theta) L_{t}-\theta t\right) \cdots \\
& \left.\exp \left(-\frac{\mu}{2}\left(Z_{t}-\delta t-z\right)-\frac{\mu^{2}}{2} \int_{0}^{t} d s Z_{s}\right) \cdot Q_{z}^{\delta}\right|_{\mathcal{F}_{t}}
\end{aligned}
$$

where we note that, for $\theta=\delta \mu / 2$, this relation simplifies as

$$
\left.Q_{z}^{\delta, \mu ; \frac{\delta \mu}{2} \downarrow}\right|_{\mathcal{F}_{t}}=\left.\frac{\exp \left(-\frac{\mu}{2} Z_{t}\right) \varphi_{\theta \downarrow}\left(Z_{t}\right)}{\exp \left(-\frac{\mu}{2} z\right) \varphi_{\theta \downarrow}(z)} \exp \left(\Psi(\theta) L_{t}-\frac{\mu^{2}}{2} \int_{0}^{t} d s Z_{s}\right) \cdot Q_{z}^{\delta}\right|_{\mathcal{F}_{t}} .
$$

The due explanation of formula (2.10) is that we have combined the "pushdownwards" formula (1.4), relative to $\left\{Q_{z}^{\delta, \mu}\right\}$, i.e. the function $\varphi_{\theta \downarrow}$ and $\Psi(\theta)$ are relative to that diffusion, with the preceding formula (2.9). From now on, we keep $\theta=\delta \mu / 2$.

We now consider what becomes of formula (2.11), once we time change both hand sides with the inverse of $\left(4 \int_{0}^{t} Z_{u} d u, t \geq 0\right)$, so that, by a slight abuse of notation, the process of reference is now $(\widehat{Z}(h), h \geq 0)$, with $\widehat{Z}$ defined by

$$
Z_{t}=\widehat{Z}\left(4 \int_{0}^{t} Z_{u} d u\right)
$$

Thus, we obtain

$$
\left.\widehat{Q}_{z}^{\delta, \mu ; \frac{\delta \mu}{2} \downarrow}\right|_{\widehat{\mathcal{F}}_{u}}=\left.\frac{\exp \left(-\frac{\mu}{2} \widehat{Z}_{u}\right) \varphi_{\theta \downarrow}\left(\widehat{Z}_{u}\right)}{\left(e^{-\frac{\mu}{2} z} \varphi_{\theta \downarrow}(z)\right)} \exp \left(\Psi(\theta) \widehat{L}_{u}-\frac{\mu^{2}}{8} u\right) \cdot \widehat{Q}_{z}^{\delta}\right|_{\widehat{\mathcal{F}}_{u}} .
$$

From the well-known property of time change for Bessel processes (see [12], Chapter XI, Proposition 1.11), $\widehat{Q}_{z}^{\delta}$ is the distribution of a Bessel process of index $\alpha / 2$, i.e. of dimension $\hat{\delta}=2-\alpha$, that is $\widehat{Q}_{z}^{\delta}=P_{z}^{\hat{\delta}}$. Again, with obvious notation, the RHS of (2.13) may be written

$$
\left.\frac{\widehat{\varphi}_{\frac{\mu^{2}}{8} \downarrow}\left(\widehat{Z}_{u}\right)}{\widehat{\varphi}_{\frac{\mu^{2}}{8} \downarrow}(z)} \exp \left(\widehat{\Psi}\left(\frac{\mu^{2}}{8}\right) \widehat{L}_{u}-\frac{\mu^{2} u}{8}\right) \cdot \widehat{Q}_{z}^{\delta}\right|_{\widehat{\mathcal{F}}_{u}},
$$

and we discover that

$$
\left\{\begin{array}{l}
\widehat{\varphi}_{\frac{\mu^{2}}{8} \downarrow}(z)=e^{-\frac{\mu}{2} z} \varphi_{\theta \downarrow}(z) \\
\widehat{\Psi}\left(\frac{\mu^{2}}{8}\right)=\Psi(\theta)
\end{array}\right.
$$


and $\widehat{Q}_{z}^{\delta, \mu ; \frac{\delta \mu}{2} \downarrow}=P_{z}^{\hat{\delta} ; \frac{\mu^{2}}{8} \downarrow}$.

Again, let us explain, very much in the same spirit, the first relation

$$
\widehat{\varphi}_{\frac{\mu^{2}}{8} \downarrow}(z)=e^{-\frac{\mu}{2} z} \varphi_{\theta \downarrow}(z)
$$

in (2.14). This translates as

$$
\widehat{E}_{z}\left(\exp \left(-\frac{\mu^{2}}{8} T_{0}(\widehat{Z})\right)\right)=e^{-\frac{\mu}{2} z} E_{z}\left(\exp \left(-\frac{\delta \mu}{2} T_{0}(Z)\right)\right),
$$

where $\widehat{Z}$ simply denotes a BES process with dimension $\widehat{\delta}$, and $Z$ a process with law $Q_{z}^{\delta, \mu}$. This may be well understood by considering the absolute continuity relationship (2.9), when we replace $t$ by $T_{0}(Z)$. Then, from that relationship, it follows that

$$
\begin{aligned}
Q_{z}^{\delta,-\mu}\left(\exp \left(-\frac{\delta \mu}{2} T_{0}(Z)\right)\right) & =e^{\frac{\mu z}{2}} Q_{z}^{\delta}\left(e^{-\frac{\mu^{2}}{2} \int_{0}^{T_{0}} d s Z_{s}}\right) \\
& =e^{\frac{\mu z}{2}} P_{z}^{\widehat{\delta}}\left(e^{-\frac{\mu^{2}}{8} T_{0}(\widehat{Z})}\right),
\end{aligned}
$$

which is precisely $(2.15)$.

Now, it is well known (see [3], [4], [10]) that the Laplace transform of $T_{0}$, under the distribution $P_{z}^{\hat{\delta}}$ of a Bessel process, is given by

$$
\widehat{\varphi}_{\frac{\mu^{2}}{8} \downarrow}(z):=E_{z}^{\hat{\delta}}\left(\exp \left(-\frac{\mu^{2}}{8} T_{0}\right)\right)=2^{1-\frac{\alpha}{2}} \Gamma\left(\frac{\alpha}{2}\right)^{-1}\left(\frac{\mu z}{2}\right)^{\alpha / 2} K_{\alpha / 2}\left(\frac{\mu z}{2}\right) .
$$

Using (2.14), we can recover the expression of $\varphi_{\theta \downarrow}$ for $\theta=\delta \mu / 2$ obtained in (??) using the identity (2.6).

\section{$\S 2.3$}

We now develop a discussion similar to the one which led to (2.8), but with the downwards arrows $\downarrow$ now changed into upwards arrows $\uparrow$; for the definition of these pushed upwards and downwards processes obtained from a diffusion, see Pitman-Yor [10]. The analogue of formula (2.10) is now

$$
\begin{aligned}
& \left.Q_{z}^{\delta, \mu ; \theta \uparrow}\right|_{\mathcal{F}_{t}} \\
= & \left.\frac{\varphi_{\theta \uparrow}\left(Z_{t}\right)}{\varphi_{\theta \uparrow}(z)} \exp (-\theta t) \exp \left(-\frac{\mu}{2}\left(Z_{t}-\delta t-z\right)-\frac{\mu^{2}}{2} \int_{0}^{t} d s Z_{s}\right) \cdot Q_{z}^{\delta}\right|_{\mathcal{F}_{t}}
\end{aligned}
$$

and we note again that, for $\theta=\delta \mu / 2$, this relation simplifies as

$$
\left.Q_{z}^{\delta, \mu ; \frac{\delta \mu}{2} \uparrow}\right|_{\mathcal{F}_{t}}=\left.\frac{\exp \left(-\frac{\mu}{2} Z_{t}\right) \varphi_{\theta \uparrow}\left(Z_{t}\right)}{\exp \left(-\frac{\mu}{2} z\right) \varphi_{\theta \uparrow}(z)} \exp \left(-\frac{\mu^{2}}{2} \int_{0}^{t} d s Z_{s}\right) \cdot Q_{z}^{\delta}\right|_{\mathcal{F}_{t}} .
$$


We note that this formula is even simpler than (2.11), since here there is no local time contribution.

We now continue to develop an analogous discussion to that made in Subsection 2.2, formula (2.8). Thus, we time-change both sides of the absolute continuity relation (2.18) with the inverse of $\left(4 \int_{0}^{t} Z_{u} d u, t \geq 0\right)$. Thus, with $\widehat{Z}$, as defined from $Z$ in $(2.12)$, we obtain

$$
\left.\widehat{Q}_{z}^{\delta, \mu ; \frac{\delta \mu}{2} \uparrow}\right|_{\widehat{\mathcal{F}}_{u}}=\left.\frac{\exp \left(-\frac{\mu}{2} \widehat{Z}_{u}\right) \varphi_{\theta \uparrow}\left(\widehat{Z}_{u}\right)}{\exp \left(-\frac{\mu}{2} z\right) \varphi_{\theta \uparrow}(z)} \exp \left(-\frac{\mu^{2} u}{8}\right) \cdot \widehat{Q}_{z}^{\delta}\right|_{\widehat{\mathcal{F}}_{u}} .
$$

With obvious notation, this RHS may be written

$$
\left.\frac{\widehat{\varphi}_{\frac{\mu^{2}}{8} \uparrow}\left(\widehat{Z}_{u}\right)}{\widehat{\varphi}_{\frac{\mu^{2}}{8} \uparrow}(z)} \exp \left(-\frac{\mu^{2} u}{8}\right) \cdot \widehat{Q}_{z}^{\delta}\right|_{\widehat{\mathcal{F}}_{u}}
$$

with

$$
\widehat{\varphi}_{\frac{\mu^{2}}{8} \uparrow}(z)=e^{-\frac{\mu}{2} z} \varphi_{\theta \uparrow}(z) .
$$

$\widehat{Q}_{z}^{\delta, \mu ; \frac{\delta \mu}{2} \uparrow}$ is the distribution of a Bessel process of dimension $\hat{\delta}=2-\alpha$ with drift $\frac{\mu^{2}}{8}$, i.e.

$$
\widehat{Q}_{z}^{\delta, \mu ; \frac{\delta \mu}{2} \uparrow}=P_{z}^{\hat{\delta} ; \frac{\mu^{2}}{8} \uparrow} .
$$

The analytical counterpart of (2.19) is the companion formula of (2.6) (see Appendix)

$$
M_{0,-\alpha / 2}(\xi)=4^{-\frac{\alpha}{2}} \Gamma\left(1-\frac{\alpha}{2}\right) \sqrt{\xi} I_{-\alpha / 2}\left(\frac{\xi}{2}\right),
$$

while the companion formula of $(2.16)$ is

$$
\widehat{\varphi}_{\frac{\mu^{2}}{8} \uparrow}(z):=\frac{1}{E_{0}^{\hat{\delta}}\left(\exp \left(-\frac{\mu^{2}}{8} T_{z}\right)\right)}=2^{-\frac{\alpha}{2}} \Gamma\left(1-\frac{\alpha}{2}\right)\left(\frac{\mu z}{2}\right)^{\frac{\alpha}{2}} I_{-\alpha / 2}\left(\frac{\mu z}{2}\right),
$$

a well-known formula which goes back to Kent [4], Pitman-Yor [10].

\section{§3. Appendix : On the Whittaker and Bessel- Macdonald Functions}

The following formulae involving these classical special functions are found in Lebedev [7], to which we refer with numberings such as : $\left(N_{*}\right) \ldots$ 
a) The Whittaker functions $M_{k, \mu}(z)$ and $W_{k, \mu}(z)$ are a pair of solutions of Whittaker's equation

$$
u^{\prime \prime}+\left(-\frac{1}{4}+\frac{k}{z}+\frac{\left(\frac{1}{4}-\mu^{2}\right)}{z^{2}}\right) u=0
$$

(p. $\left.279_{*}\right)$.

b) $W_{k, \mu}$ admits the integral representation

$$
W_{k, \mu}(z)=\frac{z^{k} e^{-\frac{z}{2}}}{\Gamma\left(\mu-k+\frac{1}{2}\right)} \int_{0}^{\infty} e^{-t} t^{\mu-k-\frac{1}{2}}\left(1+\frac{t}{z}\right)^{\mu-k+\frac{1}{2}} d t
$$

(see Problem $17_{*}$, p. $279_{*}$ ).

c)

$$
W_{0, \mu}(z)=\sqrt{\frac{z}{\pi}} K_{\mu}\left(\frac{z}{2}\right)
$$

(see Problem $19_{*}$, p. $279_{*}$ ).

d) In terms of the confluent hypergeometric functions $\Psi$, there are the relations

$$
W_{k, \mu}(z)=z^{\mu+\frac{1}{2}} e^{-\frac{z}{2}} \Psi\left(\frac{1}{2}-k+\mu, 2 \mu+1 ; z\right)
$$

$\left(\right.$ see $(9.13 .16)_{*}$, p. $\left.274_{*}\right)$,

$$
K_{\mu}(z)=\sqrt{\pi}(2 z)^{\mu} e^{-z} \Psi\left(\mu+\frac{1}{2}, 2 \mu+1 ; 2 z\right)
$$

$\left(\right.$ see $(9.13 .15)_{*}$, p. $\left.274_{*}\right)$.

Thus, taking $k=0$ in the above formula for $W_{k, \mu}$, one recovers c).

e) In terms of the confluent hypergeometric functions $\Phi$, there are the relations

$$
M_{k, \mu}(z)=z^{\mu+\frac{1}{2}} e^{-\frac{z}{2}} \Phi\left(\frac{1}{2}-k+\mu, 2 \mu+1 ; z\right)
$$

$\left(\right.$ see $(9.13 .16)_{*}$, p. $\left.274_{*}\right)$,

$$
I_{\mu}(z)=\frac{(z / 2)^{\mu}}{\Gamma(\mu+1)} e^{-z} \Phi\left(\mu+\frac{1}{2}, 2 \mu+1 ; 2 z\right)
$$

$\left(\right.$ see $(9.13 .14)_{*}$, p. $\left.274_{*}\right)$.

Thus, taking $k=0$ in the above formula for $M_{k, \mu}$, we obtain

$$
M_{0, \mu}(z)=4^{\mu} \Gamma(\mu+1) \sqrt{z} I_{\mu}\left(\frac{z}{2}\right) .
$$




\section{References}

[1] C. Donati-Martin and M. Yor, Some explicit Krein representations of certain subordinators, including the Gamma process, Publ. Res. Inst. Math. Sci. 42 (2006), 879-895.

[2] C. Donati-Martin, B. Roynette, P. Vallois and M. Yor, On constants related to the choice of the local time at 0 , and the corresponding Itô measure for Bessel processes with dimension $d=2(1-\alpha), 0<\alpha<1$, to appear in Studia Math. Hung., vol. 44, no. 4, (2007).

[3] R. K. Getoor, The Brownian escape process, Ann. Probab. 7 (1979), no. 5, 864-867.

[4] J. Kent, Some probabilistic properties of Bessel functions, Ann. Probab. 6 (1978), no. 5, $760-770$.

[5] F. B. Knight, Characterization of the Lévy measures of inverse local times of gap diffusion, in Seminar on Stochastic Processes, 1981 (Evanston, Ill., 1981), 53-78, Birkhäuser, Boston, Mass.

[6] S. Kotani and S. Watanabe, Kreĭn's spectral theory of strings and generalized diffusion processes, in Functional analysis in Markov processes (Katata/Kyoto, 1981), 235-259, Lecture Notes in Math., 923, Springer, Berlin.

[7] N. N. Lebedev, Special Functions and their Applications, Revised edition, translated from the Russian and edited by Richard A. Silverman, Dover, New York, 1972.

[8] S. A. Molčanov and E. Ostrovskǐr, Symmetric stable processes as traces of degenerate diffusion processes, Teor. Verojatnost. i Primenen. 14 (1969), 127-130.

[9] J. Pitman and M. Yor, A decomposition of Bessel bridges, Z. Wahrsch. Verw. Gebiete 59 (1982), no. 4, 425-457.

[10] Bessel processes and infinitely divisible laws, in Stochastic integrals (Proc. Sympos., Univ. Durham, Durham, 1980), 285-370, Lecture Notes in Math., 851, Springer, Berlin.

[11] abilités, XXXI, 272-286, Lecture Notes in Math., 1655, Springer, Berlin.

[12] D. Revuz and M. Yor, Continuous martingales and Brownian motion. Third edition. Grundlehren der Mathematischen Wissenschaften [Fundamental Principles of Mathematical Sciences], 293. Springer-Verlag, Berlin, 1999. xiv+602 pp.

[13] M. Yor, On certain exponential functionals of real-valued Brownian Motion. Paper $\sharp 1$ in: Exponential Functionals of Brownian motion and related processes. Springer-Finance (2001). Translated from: Sur certaines fonctionnelles exponentielles du mouvement brownien réel. J. Appl. Prob. 29 (1992), 202-208. 